\title{
Síndrome de intestino irritable en pacientes con síndrome dispéptico menores de 55 años
}

\author{
L. BUJANDA, I. URIARTE, C. MUÑOZ, A. SÁNCHEZ, C. IRIONDO, A. GIL- \\ MOLET, M. E. ALKIZA ${ }^{1}$ \\ Servicio de Digestivo. Hospital de San Eloy. Baracaldo. Vizcaya \\ ${ }^{1}$ Departamento de Salud Pública del Gobierno Vasco
}

IRRITABLE BOWEL SYNDROME IN PATIENTS UNDER AGE 55 WITH DYSPEPTIC SYNDROME

\begin{abstract}
RESUMEN
Se estudió la presencia de síndrome intestino irritable (SII) en 289 pacientes menores de 55 años con síndrome dispéptico (SD). El número de pacientes diagnosticados de SII fue 49 (17\%).El grupo I se consideró a los pacientes con SD (240 pacientes), mientras que el grupo II eran los pacientes con SD y SII (49 pacientes). El grupo II acudió con más frecuencia a las consultas y antes ( $92 \%$ volvio a las consultas y tardaron 27 meses en volver) que los pacientes del grupo I (52\% y 40 meses) $(\mathrm{p}<0,05)$. El número de pruebas diagnósticas solicitadas fue superior en los pacientes del grupo II. La patología psiquiátrica fue más frecuente en el grupo II que en el grupo I (20\% frente al $7 \%)(\mathrm{p}<0,05)$. Los pacientes con SD que son diagnosticados de SII, acuden con más frecuencia y antes a la consulta. El número de pruebas diagnósticas solicitadas por paciente es mayor en los pacientes con SII y SD.
\end{abstract}

PALABRAS CLAVE: Pronóstico. Síndrome de intestino irritable. Síndrome dispéptico. Jóvenes.

\begin{abstract}
The presence of IBS was studied in 289 patients under 55 years of age with dyspeptic syndrome. The subjects were divided into two groups: group I (240 patients with DS) and group II (49 patients with DS and IBS). The mean duration of follow-up was 10.8 years (130 months). IBS was diagnosed in 49 patients $(17 \%)$. Females accounted for $64 \%$ and $77 \%$ of groups I and II, respectively $(p<0.05)$. There were no differences in mean age or the type of FDS. The patients in group II consulted more often and earlier $(92 \%$ returned for consultation, and took 27 months in doing so) than those in group I ( $52 \%$ and 40 months $)(p<0.05)$. The number of diagnostic tests requested was greater in group II. Psychiatric pathology was in turn more common in group II than in group I (20\% versus $7 \%)(p<0.05)$.
\end{abstract}

KEY WORDS: Prognosis. Irritable bowel syndrome. Functional dyspeptic. Young.

Bujanda L, Uriarte I, Carmen Muñoz, Sánchez A, Iriondo C, Gil-Molet A, Alkiza ME. Síndrome de intestino irritable en pacientes con síndrome dispéptico menores de 55 años. An Med Interna (Madrid) 2003; 20: 360-362.

\section{INTRODUCCIÓN}

El síndrome de intestino irritable (SII) es un trastorno funcional, caracterizado por dolor y alteración del ritmo intestinal asociado con síntomas de hinchazón y meteorismo. Estudios en varios países industrializados indican una prevalencia entre un 15 a un $20 \%$, una mayor prevalencia en el sexo femenino y que muchos de ellos no buscan atención médica por sus síntomas (1-3).

El SII se ha asociado con otros trastornos funcionales, como el síndrome dispéptico (SD). Se ha estimado que al menos un tercio de los pacientes con SD tienen SII. Mas aún, utilizando mediciones estandarizadas para valorar los síntomas se observa como muchos pacientes con SD tienen síntomas compatibles con varios subtipos de dispepsia y con síntomas de SII $(4,5)$. La etiopatogenia del SII y del SD son desconocidos y se piensa que ambos pueden estar relacionados.

Nuestro objetivo era valorar la prevalencia de SII en pacientes con síndrome dispéptico menores de 55 años atendi- dos en la consulta de digestivo a lo largo de más de 10 años y sus características.

\section{CASOS APORTADOS}

Se estudia la prevalencia de SII en 307 pacientes menores de 55 años con el diagnóstico inicial de dispepsia atendidos por primera vez antes de 1995 en la consulta extrahospitalaria de aparato digestivo del Hospital San Eloy de Baracaldo. Este hospital constituye un centro sanitario secundario para una población aproximada de 120.000 habitantes. Desde su puesta en funcionamiento (1956) hasta la actualidad (septiembre de 1999) se han tratado 20.000 pacientes con enfermedades digestivas, enviados o derivados desde los centros de asistencia primaria de Baracaldo y zonas limítrofes.

Se revisaron 2.500 historias clínicas de la consulta para la selección de los 307 pacientes $(12 \%)$. Se incluyeron los sujetos que cumplían los siguientes criterios de inclusión: dolor o molestia abdominal localizado en el abdomen superior durante al menos tres meses,

Trabajo aceptado: 17 de diciembre de 2002

Correspondencia: Luis Bujanda. C/ Ocharán Mazas, 13, Q-1º A. 39700 Castro Urdiales. e-mail: digestivo.saneloy\&hsel.osakidetza.net. 
la no existencia de antecedentes de cirugía gástrica, intestinal, biliar o pancreática ni historia previa de enfermedad ulcerosa y ausencia de signos y síntomas de alarma.

Entre los signos y síntomas de alarma se consideraron la presencia de anorexia, anemia, pérdida de peso, odinofagia, disfagia, masa epigástrica, vómitos de repetición o de retención e ictericia. Fueron excluídos los pacientes que tomaban antiinflamatorios no esteroideos (AINES), los pacientes con clínica ulcerosa típica (dolor epigástrico periódico que mejora con antiácidos y/o la comida), los pacientes con síntomas evidentes de reflujo, los pacientes con anormalidades en la analítica básica (glucosa, creatinina, enzimas hepáticas, urea, hemograma y velocidad de sedimentación), los pacientes con síntomas sugestivos de cólico biliar y las mujeres embarazadas.

El síndrome dispéptico se clasificó en subtipos según los criterios de Tallley y cols (6) en:

Tipo ulcus; pacientes con dolor o molestia en epigastrio, a veces exagerado para el estado general del paciente, difuso, cambiante, con irradiación anómala, sin clara mejoría con antiácidos, ni clara relación con la comida.

Tipo dismotilidad; pacientes con molestia o dolor en epigastrio y al menos algunos de los siguientes síntomas que sugiere trastorno en la motilidad del tracto digestivo superior como 1 , naúseas o vómitos, 2 hinchazón y distensión abdominal visible, 3 pesadez precoz

Tipo mixto; pacientes con síntomas de los dos tipos anteriores sin predominar ninguno de ellos o aquellos que es difícil de encuadrarlos en los anteriores.

El síntoma de reflujo sólo era considerado cuando el paciente presentaba episodios de pirosis o regurgitación ácida pero además presentaba síntomas de dispepsia tipo dismotilidad o tipo ulcus-like, y en esos casos se consideraba como mixto.

Se excluyeron los pacientes que presentaron patología orgánica en el estudio gastroduodenal o esofagogastroscopia en la primera visita.

Para el diagnóstico de SII, el paciente debía cumplir los criterios de Roma (7), tener al menos dos coprocultivos y parasitos en heces negativos y tener un enema opaco o una rectosigmoidoscopia normales.

Se consideraron dos grupos; el grupo I los pacientes diagnosticados de SD y el grupo II los pacientes con SD y SII.

En todos los pacientes incluidos se analizó la edad en que fue a la consulta por primera vez, el tipo de estudio realizado en la primera consulta (estudio gastroduodenal baritado o endoscopia), el tipo de $\mathrm{SD}$, el número de consultas posteriores realizadas hasta Setiembre de 1999, las pruebas realizadas, el diagnóstico realizado en cada uno de ellas, el tiempo transcurrido hasta el diagnóstico de SII y la asociación con enfermedades psiquiátricas. El tiempo de seguimiento comprendía el periodo entre la primera consulta y Setiembre de 1999. El tratamiento no fue considerado debido a la gran diversidad de los mismos, a los diferentes tiempos utilizados y a la falta de información sobre la respuesta a los mismos.

Las comparaciones entre grupos se realizó según los casos con los test $\chi^{2}$ de Yates corregido, test de Fisher ó el test de Mann-Whitney. Las variables continuas eran comparadas por la t de Student o por el test $U$ Mann-Whitney. Una p inferiror o igual a 0,05 era considerada como estadísticamente significativa.

Las pruebas realizadas en la primera consulta fueron un estudio gastroduodenal baritado en 249 pacientes, una esofagogastroscopia en 22 y en 26 no se realizó ninguna de las dos pruebas anteriores.

De todos los pacientes a los que se realizó alguna exploración complementaria en la primera visita (estudio gastroduodenal o esofagogastroscopia) se encontró patología orgánica en 18, en 10 pacientes con el estudio baritado gastroduodenal y en 8 con la esofagogastroscopia.

En total se incluyeron 289 pacientes con diagnóstico inicial de dispepsia. El tiempo medio de seguimiento fue de 130 meses (10,8 años). De ellos en 49 (17\%) pacientes se diagnóstico síndrome de intestino irritable. Las características generales de cada grupo se reflejan en la Tabla I.
TABLA I

CARACTERÍSTICAS GENERALES DE LOS PACIENTES

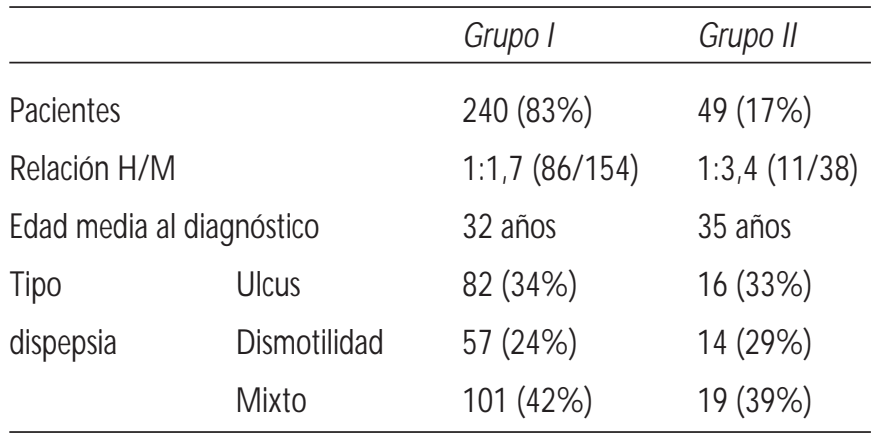

De los 240 pacientes del grupo I volvieron a consultar por sus molestias $125(52 \%)$ y el tiempo medio que tardaron en consultar de nuevo fue de 40 meses. Entre los 49 pacientes del grupo II volvieron a consultar $45(92 \%)$ y el tiempo medio en realizar la segunda consulta fue de 27 meses. Cuando consideramos el mismo tiempo de seguimiento para todos los pacientes (60 meses) observamos como los pacientes del grupo II volvían más a las consultas y más precozmente que los pacientes del grupo I $(\mathrm{p}<0,05)$.

El tiempo medio que se tardó en realizar el diagnóstico de SII fue de 77 meses (Fig. 1). En 6 pacientes se realizó a la vez que el SD.

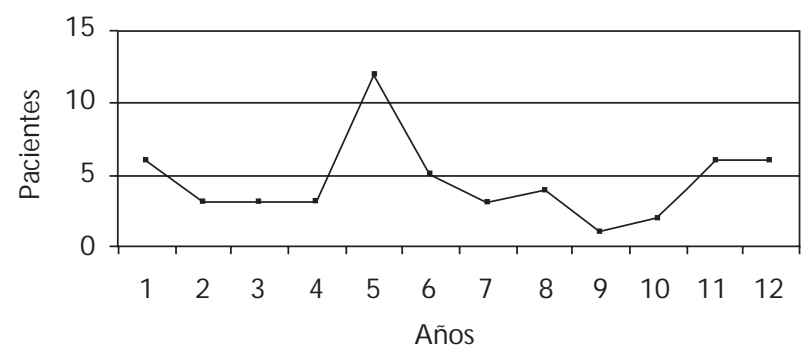

Fig. 1. Diagnóstico del síndrome de intestino irritable a lo largo de los años a pacientes con síndrome dispéptico.

Se diagnóstico patología psiquiátrica en 27 pacientes, 10 (20\%) en el grupo II y 17 (7\%) en los pacientes del grupo I. La patología psiquiátrica diagnosticada fue en 11 pacientes trastornos de la ansiedad, en 8 depresión, en 6 trastornos ansioso-depresivos, en un paciente anorexia nerviosa y en otro un trastorno psiquiátrico no definido.

El número de pruebas que se realizó en ambos grupos a lo largo de su evolución están representados en la Tabla II. El número de pruebas diagnósticas solicitadas para un mismo tiempo de seguimiento (60 meses) fue superior en los pacientes del grupo II (1,42 pruebas por paciente frente a un 0,64$)(\mathrm{p}<0,05)$.

\section{DISCUSIÓN}

Los síntomas y las alteraciones que afectan a los trastornos funcionales digestivos se caracterizan por afectar a la musculatura lisa de tracto gastrointestinal y por alteraciones en la percepción visceral $(8,9)$. La prevalencia de SII en pacientes con SD es mayor que en la población general, entre un $23 \%$ y un $50 \%(10,11)$, y es más frecuente en mujeres (12). En nues- 
TABLA II

PRUEBAS DIAGNÓ STICAS SO LICITADAS SEGÚN LA PRESENCIA O NO DE SII

\begin{tabular}{|c|c|c|}
\hline & $\begin{array}{l}\text { Grupo I } \\
\text { (no de pruebas } \\
\text { por pacientes) }\end{array}$ & $\begin{array}{l}\text { Grupo II } \\
\text { (no de pruebas } \\
\text { por pacientes) }\end{array}$ \\
\hline Estudio gastroduodenal & $91(0,38)$ & $55(1,15)$ \\
\hline Esofagogastroscopia & $125(0,52)$ & $39(0,81)$ \\
\hline Colecistografía oral & $11(0,05)$ & $15(0,31)$ \\
\hline Ecografía abdominal & $45(0,19)$ & $39(0,81)$ \\
\hline Enema opaco & $23(0,1)$ & $43(0,90)$ \\
\hline Rectosigmoidoscopia & $8(0,03)$ & $14(0,29)$ \\
\hline Colonoscopia & $3(0,01)$ & $3(0,06)$ \\
\hline Tránsito intestinal & $3(0,01)$ & $5(0,10)$ \\
\hline TAC & $1(0,005)$ & $2(0,04)$ \\
\hline Total & 310 & 215 \\
\hline
\end{tabular}

SD; SII;

tra serie la prevalencia fue inferior $(17 \%)$ probablemente debido a que era un estudio retrospectivo en el que no se buscaba expresamente los síntomas del SII y a que se exigió que tuvieran los criterios de Roma, al menos dos coprocultivos y parásitos negativos y un enema opaco o una colonoscopia normales (10).

Algunos autores sugieren que los pacientes con SD y SII deberían incluirse in el subgrupo de pacientes con dispepsia tipo dismotilidad debido a que muchos de estos pacientes presentan anormalidades motoras gastrointestinales $(13,14)$. Sin embargo, en nuestro estudio y otros (10) la distribución de los pacientes con SD era similar entre los pacientes con o sin SII.

Una característica que diferencia los pacientes con SII de las personas sanas y de otros grupos de pacientes es que estos pacientes tienen respuestas fisiológicas y sintomáticas mayores ante situaciones estresantes y con más frecuencia son diagnósticados de enfermedades psiquiátricas como pueden ser trastornos de la personalidad, ansiedad, depresión y tendencia a la somatización (1). Sin embargo, no existe un patrón psicopatológico en estos pacientes característico. Nosotros observamos como los pacientes con SII y SD tenían más enfermedades psiquiátricas sin observar un tipo de enfermedad psiquiátrica característica. Esta relación puede ser debida a que estos pacientes tengan un trastorno funcional más severo que les produzca alteraciones psicopatológicas, o al revés, pacientes con trastornos psicopatológicos tiendan a somatizar más en diferentes localizaciones del aparato gastrointestinal. La exactitud de esta interrelación actualmente es desconocida.

El diagnóstico de los trastornos funcionales digestivos, en ocasiones, es difícil. En parte debido a que no existe ninguna prueba diagnóstica específica de estas enfermedades, por lo que, el número de pruebas diagnósticas que se pueden realizar es muy grande. Así se ha observado como los pacientes con trastornos funcionales digestivos más severos tienen más riesgo de realizar más pruebas diagnósticas innecesarias (15). En nuestro estudio, los pacientes que presentaron SII y SD consultaron con más frecuencia y antes que los pacientes con sólo SD. También el número de pruebas diagnósticas que se solicitaron fue mayor en el grupo con SII y SD. Es posible que los pacientes con SII y SD tengan síntomas funcionales más severos, en ocasiones asociados a trastornos psicopatológicos, que les haga recaer con más frecuencia y volver antes a las consultas. Por estos mismos motivos y probablemente a que son pacientes más demandantes, el gastroenterólogo tiene que recurrir con más frecuencia a pruebas diagnósticas que aseguren su diagnóstico, y a la vez, tranquilizen al paciente.

\section{Bibliografía}

1. Drossman DA, Thompson WG. The irritable bowel syndrome: review and a graduated multicomponent treatment approach. Ann Intern Med 1992; 116: 1009-16.

2. Thompson WG, Heaton KW, Smyth GT, Smyth C. Irritable bowel syndrome in general practice: prevalence, characteristics, and referral. Gut 2000; 46: 78-82.

3. Jones R. IBS: prime problem in primary care. Gut 2000; 46: 7-8.

4. Holtmann G, Goebell H, Talley NJ. Functional dyspepsia and irritable bowel syndrome. Am J Gastroenterol 1997; 92: 954-9.

5. Agréus L, Svardsudd K, Nyrén O, Tibblin G. Irritable bowel syndrome and dyspepsia in the general population: overlap and lack of stability over time. Gastroenterolgy 1995; 109: 671-680.

6. Talley NJ, Stanghellini V, Heading RC, Koch KL, Malagelada JR, Tytgat GNJ. Functional gastroduodenal disorders. Gut 1999; 45 (Supl. II): II37-II42.

7. Thompson WG, Longstreth GF, Drossman DA, Heaton KW, Irvine EJ, Muller-Lissner SA. Functional bowel disorders and functional abdominal pain. Gut 1999; 45 (Supl. II): II43-7.

8. Trimble KC, Farouk R, Pryde A, Douglas S, Heading RC. Heightened visceral sensation in funcional gastrointestinal disease is not site-speci- fic: Evidence for a generalized disorder of gut sensitivity. Dig Dis Sci 1995; 40: 1607-13.

9. Mayer EA, Gebhart GF. Basic and clinical aspects of visceral hyperalgesia. Gastroenterology 1994; 107:271-93.

10. American Gastroenterological Association medical position statement: irritable bowel syndrome. Gastroenterology 1997; 112: 2118-37.

11. Holtman G, Goebell H, Talley NJ. Dyspepsia in consulters and nonconsulters: Prevalence, health-care seeking behaviour and risk factors. Eur J Gastroenterol Hepatol 1994; 6: 917-24.

12. Schlemper RJ, van der Werf SD, Vandenbroucke JP, Biemond I, Lamers CB. Peptic ulcer, non-ulcer dyspepsia and irritable bowel syndrome in The Netherlands and Japan. Scand J Gastroenterol 1993; 200 (Supl.): 33-41.

13. Holtmann G, Goebell H, Talley NJ. Functional dyspepsia and irritable bowel syndrome: is there a common pathophysiological basis?. Am J Gastroenterol 1999; 92: 954-9.

14. Colin-Jones DG, Bloom B, Bodemar G, et al. Management of dyspepsia: report of a working party. Lancet 1988; 1: 576-9.

15. Drossman DA. Illness behaviour in the irritable bowel syndrome. Gastroenterol Int 1991; 4: 77-81. 